EPJ Web of Conferences 85, 02010 (2015)

DOI: $10.1051 /$ epjconf/ 20158502010

(C) Owned by the authors, published by EDP Sciences, 2015

\title{
Chiral Odd GPDs
}

\author{
Gary R. Goldstein ${ }^{1, a}$, J. Osvaldo Gonzalez Hernandez ${ }^{2, b}$, and Simonetta Liuti ${ }^{3, c}$ \\ ${ }^{1}$ Department of Physics and Astronomy, Tufts University, Medford, MA 02155, US \\ ${ }^{2}$ Istituto Nazionale di Fisica Nucleare (INFN) - Sezione di Torino via P. Giuria, 1, 10125 Torino, ITALY, Italy \\ ${ }^{3}$ University of Virginia - Physics Department, 382 McCormick Rd., Charlottesville, Virginia 22904 - USA \\ and Laboratori Nazionali di Frascati, INFN, Frascati, Italy.
}

\begin{abstract}
Nucleon spin structure, transversity and the tensor charge are of central importance to understanding the role of QCD in hadronic physics. A new approach to measuring orbital angular momenta of quarks in the proton via twist 3 GPDs is shown. The "flexible parametrization" of chiral even GPDs is reviewed and its transformation into the chiral odd sector is discussed. The resulting parametrization is applied to recent data on $\pi^{0}$ and $\eta$ electroproduction.
\end{abstract}

\section{Introduction}

Understanding the nature of angular momenta among the quark and gluon fields that compose the nucleon has been of great interest for decades. Recently there has been considerable attention focused on the interpretation of Orbital Angular Momentum (OAM) within the constraints of gauge invariance and Lorentz covariance. We will briefly present a method for measuring the twist 3 GPD $G_{2}$ that is associated with "dynamical OAM" [1]. Then we will summarize the "flexible parametrization" for chiral even, leading twist GPDs that have been applied extensively to DVCS [2] and EM form factor data [3]. Using the helicity representation for GPDs, the chiral odd sector normalizations are nearly determined [4]. The resulting observables are compared to experimental data.

Why are chiral odd GPDs important? For one thing, they are related to the transversity distribution of the fields in the polarized nucleon, $h_{1}(x)$ [5]. That distribution, which gives this workshop its name [6], is on an equal footing with the helicity distribution, $g_{1}(x)$, and contains as much information about spin and angular momentum in the soft QCD regime. The importance of that transversity distribution has long been recognized [7]. However, accessing transversity is hampered by its connection to chiral odd interactions, so that it has to be extracted from semi-inclusive or exclusive processes. The experiments and the modeling have been underway for quite a while. The first moment of the transversity distribution is the tensor charge, $\delta q$, for which there are predictions from model calculations for the soft QCD physics and some extractions from SIDIS data, shown in Fig. 1. We will present

\footnotetext{
a e-mail: gary.goldstein@ tufts.edu

be-mail: joghdr@gmail.com

ce-mail: sl4y@virginia.edu
}

our own determinations [8], based on the modeling and parametrizing we have examined.

\section{Orbital Angular Momentum - Measurements}

Recent work interpreting OAM, summarized in E. Leader's presentation [9], has led us to consider higher twist contributions, as encoded in GPDs or Generalized Transverse Momentum Distributions (GTMDs). From the Ji sum rule [10] expressing quark OAM, Ref. [11] showed that the OAM arose as the first moment of a twist 3 GPD, $G_{2}(x, \xi, \Delta)$. Specifically,

$$
\begin{array}{r}
\int d x x G_{2}^{q}(x, 0,0)=\frac{1}{2}\left[-\int d x x\left(H^{q}(x, 0,0)\right.\right. \\
\left.\left.+E^{q}(x, 0,0)\right)+\int d x \tilde{H}^{q}(x, 0,0)\right]
\end{array}
$$

We have shown [1] that the left side can be obtained from DVCS electroproduction data for a longitudinally polarized target (with respect to the virtual photon direction), while the twist 2 GPDs on the right side can be obtained from pdf's and independent measurements. More precisely, the longitudinal asymmetry involves the interference between the GPD handbag amplitudes, including twist $3 G_{2}$, and the Bethe-Heitler amplitudes [12]. The corresponding asymmetry $A_{U L}$ can be decomposed into azimuthal modulations

$$
A_{U L}=\frac{a \sin \phi+b \sin 2 \phi}{c_{0}+c_{1} \cos \phi+c_{2} \cos 2 \phi}
$$

where $\phi$ is the azimuthal angle of the hadron plane relative to the lepton plane. The $\sin \phi$ is a twist 2 term (in the interference between the GPD and the Bethe-Heitler 
amplitudes), while the $\sin 2 \phi$ term is proportional to the twist $3 G_{2}$. Fig. 2 shows the result of evaluating the two asymmetries in our model for the GPDs, having used the Wandzura-Wilczek approximation for the twist 3 GPD. The near agreement with HERMES data [13] is quite encouraging for obtaining OAM from asymmetry measurements.

\section{Chiral even "flexible parametrization"}

The four quark chiral even GPDs, $H, E, \widetilde{H}, \widetilde{E}$ are dependent on kinematic variables $(x, \xi, t)$, and evolve in $Q^{2}$. These GPDs are constrained in several ways - first by DIS data and by pdf's, $H^{q}(x, 0,0)=q(x)$ and $\widetilde{H}^{q}(x, 0,0)=$ $\Delta q(x)$. The first moments of $H$ and $E$ are the EM form factors $F_{1}(t)$ and $F_{2}(t)$, respectively. The first moments of $\widetilde{H}^{q}(x, \xi, t)$ and $\widetilde{E}^{q}(x, \xi, t)$ are the axial form factors $G_{A}^{q}(t)$ and the pseudoscalar form factor $G_{P}^{q}(t)$. We applied a recursive procedure to match these constraints, systematically, and to provide a flexible parametrization [2]. We developed a model for the GPDs that has spectator scalar and axial vector diquarks. Regge behavior arises from a spectral distribution of spectator diquark masses [14-16], leading to a hybrid form [2], generically, for any of the four GPDs

$$
F(X, \zeta, t)=\mathcal{N} G_{M_{X}, m}^{M_{\Lambda}}(X, \zeta, t) R_{p}^{\alpha, \alpha^{\prime}}(X, \zeta, t)
$$

where the Regge factor is

$$
R_{p}^{\alpha, \alpha^{\prime}}(X, \zeta, t)=X^{-\left[\alpha+\alpha^{\prime}(X) t+\beta(\zeta) t\right]}
$$

while $G$ has the diquark form. The latter can be written in terms of a proton-quark-diquark vertex, with a form factor, or helcity dependent light front wave functions for the incoming states convoluted with the corresponding outgoing states as illustrated in Fig. 3

The overall helicity amplitudes for DVCS factorize, via a handbag picture, into the hard part $\left(\gamma^{*}+\right.$ quark $\rightarrow \gamma+$ quark $^{\prime}$ ) and the soft, GPD part ( $p \rightarrow$ quark : quark $\rightarrow p^{\prime}$ ). The GPD contribution has been expressed in terms of helicity amplitudes $A_{\Lambda_{p}^{\prime}, \lambda^{\prime} ; \Lambda_{p}, \lambda}$, with helicity labels from the outgoing nucleon, the returning quark, incoming nucleon, outgoing quark, respectively.

This model leads to predictions for measured processes. One example of the data match (from JLab Hall A) with this model is the beam polarization asymmetry, shown in Fig. 10. Another example, the beam charge asymmetry, is shown in Fig. 4 and compared with HERMES data. For many such predictions, see [2].

Recent data on EM form factors [19] have been used to make more precise flavor separation for the GPDs and their first moments [3]. The form factor data and our model results are shown in Fig. 5. With our well tuned chiral even model for $H^{q}(x, 0,0)$ and $E^{q}(x, 0,0)$ we obtain quark angular momenta from Ji's sum rule [10],

$$
J_{q}=\frac{1}{2} \int_{-1}^{1} d x x\left(H_{q}\left(x, 0,0 ; Q^{2}\right)+E_{q}\left(x, 0,0 ; Q^{2}\right)\right),
$$

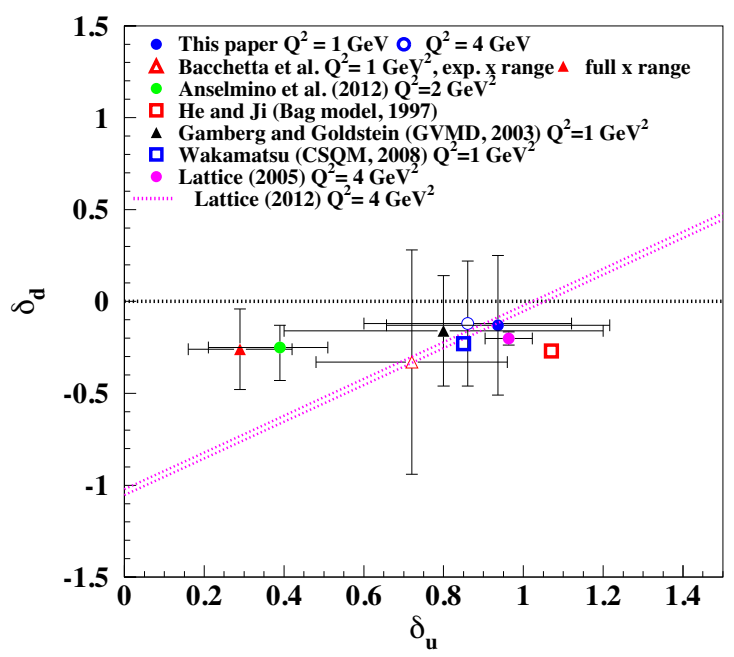

Figure 1. Predictions and determinations of tensor charges $\delta_{u}, \delta_{d}$, adapted from Ref. [8].

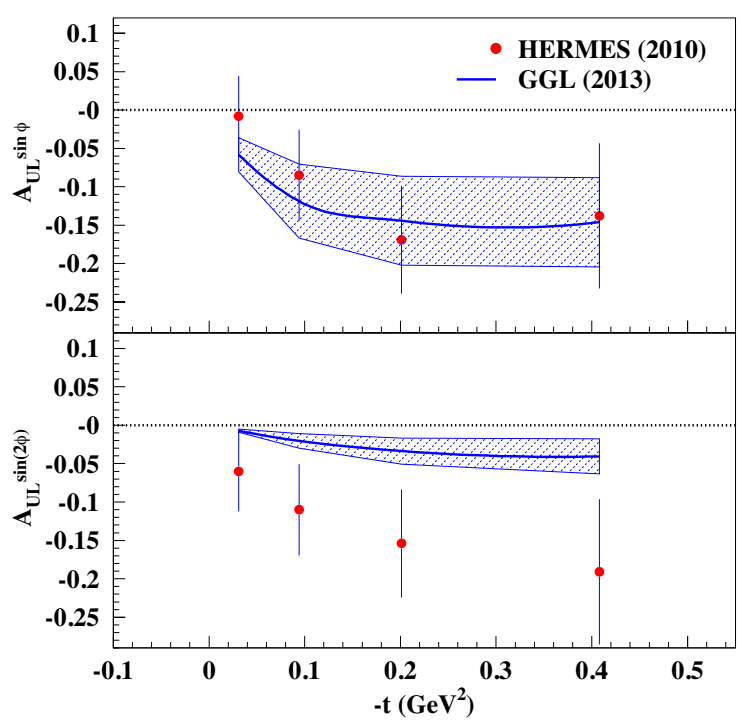

Figure 2. The asymmetry $A_{U L}$ twist two $(\sin \phi)$ and twist three $(\sin 2 \phi)$ modulations plotted vs. $-t$, compared to HERMES data [13] at $x_{B j}$ and $Q^{2}$ of the data. The blue bands are predictions from GPD model of [2,3]. Adapted from Ref. [1].

and the quarks orbital angular momentum obtained as,

$$
L_{q}=J_{q}-\frac{1}{2} \int_{-1}^{1} d x \tilde{H}_{q}\left(x, 0,0 ; Q^{2}\right)
$$

where $Q^{2}$ is the process' scale. Our results obtained evolving all GPDs at leading order [2] are shown in Figures 6,7. 


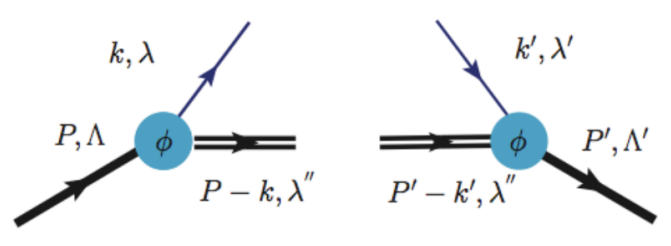

Figure 3. Vertex structures defining the spectator model tree level diagrams.

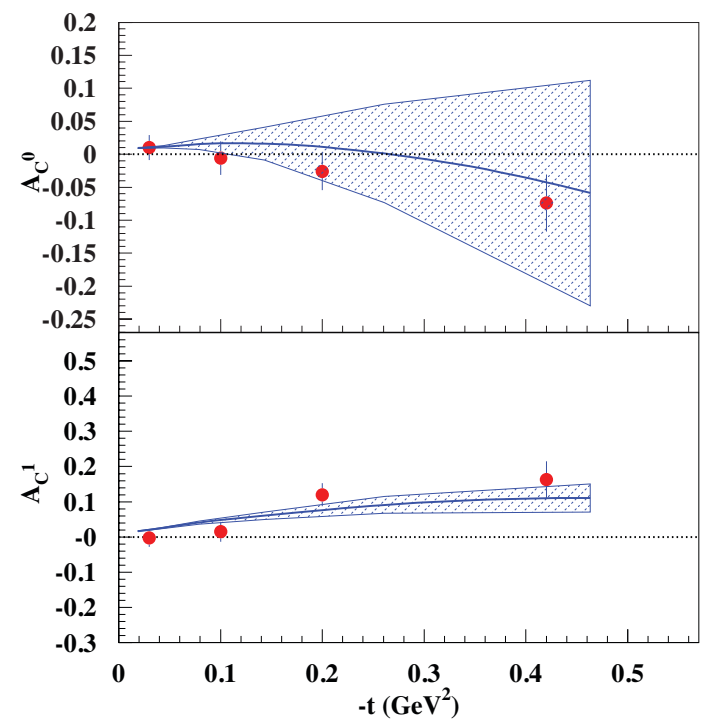

Figure 4. Coefficients of the beam charge asymmetry, $A_{C} \propto A_{C}^{0}+$ $A_{C}^{1} \cos \phi$, extracted from experiment $[17,18]$. The lower panel is the coefficient for the $\cos \phi$ dependent term, while the upper panel is the $\cos \phi$ independent term. Adapted from Ref. [1].

\section{Chiral odd sector}

The chiral odd GPDs are $H_{T}, E_{T}, \widetilde{H}_{T}, \widetilde{E}_{T}$. Of particular interest is $H_{T}(x, \xi, t)$, which gives the Transversity distribution, $H_{T}(x, 0,0)=h_{1}(x)$. Starting all over to obtain a viable model for these chiral odd GPDs would leave us with few constraints on the normalizations and $t, \xi$ dependences. However, because we developed a successful model for the chiral even sector we can use a simple parity relation to obtain chiral odd GPDs. Simply put, consider the scalar diquark model for the helicity amplitudes illustrated in Fig. 3. Each side corresponds to a function $\phi_{\Lambda, \lambda}$ to form

$$
A_{\Lambda^{\prime} \lambda^{\prime}, \Lambda \lambda}^{(0)}=\int d^{2} k_{\perp} \phi_{\Lambda^{\prime} \lambda^{\prime}}^{*}\left(k^{\prime}, P^{\prime}\right) \phi_{\Lambda \lambda}(k, P),
$$

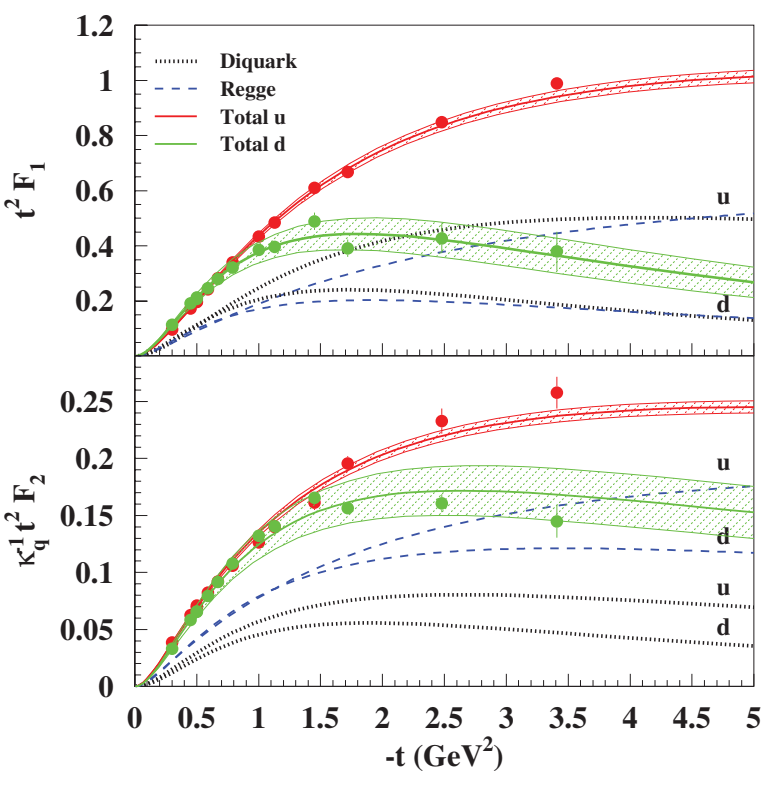

Figure 5. $t^{2} F_{1}^{q}, q=u, d$ (above) and $\kappa_{q}^{-1} t^{2} F_{2}^{q}$ (below) plotted vs. $-t$, from our parametrization [3]. Data from Ref.[19]. Dotted $=$ diquark Eq.(3); dashed = Regge Eq.(4), adapted from Ref. [3].

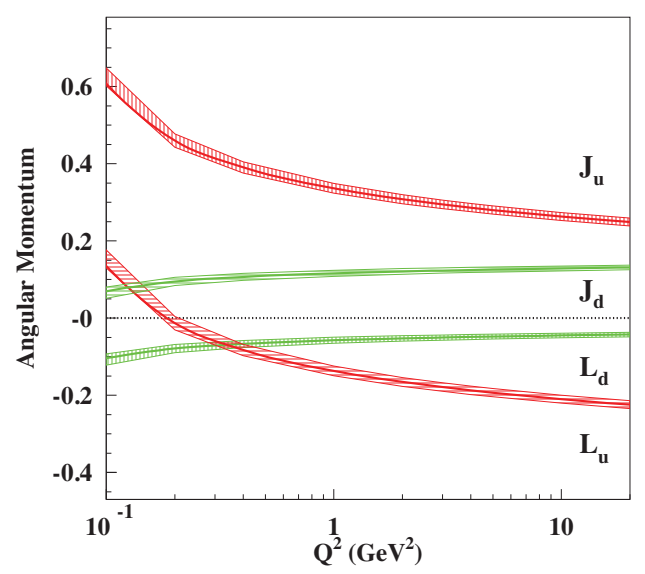

Figure 6. Quarks angular momentum, $J_{q}$, and orbital angular momentum, $L_{q}$, plotted vs. the scale $Q^{2}$. Adapted from Ref. [3]

with vertex structures

$$
\begin{aligned}
\phi_{\Lambda, \lambda}(k, P) & =\Gamma(k) \frac{\bar{u}(k, \lambda) U(P, \Lambda)}{k^{2}-m^{2}} \\
\phi_{\Lambda^{\prime} \lambda^{\prime}}^{*}\left(k^{\prime}, P^{\prime}\right) & =\Gamma\left(k^{\prime}\right) \frac{\bar{U}\left(P^{\prime}, \Lambda^{\prime}\right) u\left(k^{\prime}, \lambda^{\prime}\right)}{k^{\prime 2}-m^{2}} .
\end{aligned}
$$

where we defined the proton-quark-diquark coupling as,

$$
\Gamma=g_{s} \frac{k^{2}-m_{q}^{2}}{\left(k^{2}-M_{\Lambda}^{q^{2}}\right)^{2}} .
$$




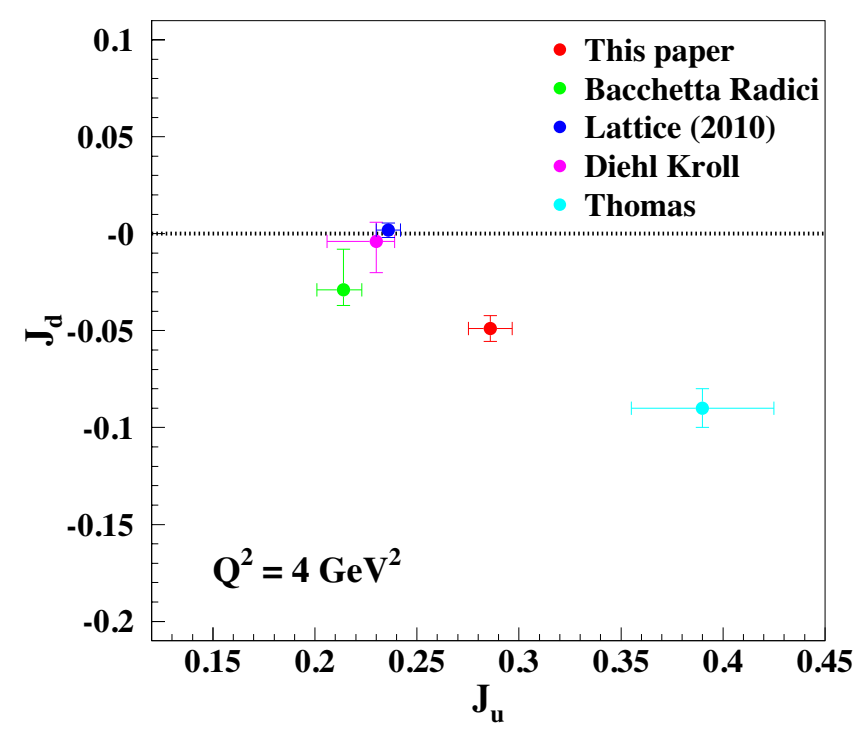

Figure 7. $J_{u}$ vs. $J_{d}$ obtained from our Ref. [2, 3] as constrained by flavor separated Dirac and Pauli form factors, compared to other determinations. Adapted from Ref. [3].

A very similar form follows for axial vector diquarks, although complicated by the matching of the diquark helicities from left to right.

The Parity relations for the vertices in Fig.3 read,

$$
\phi_{-\Lambda-\lambda}=(-1)^{\Lambda-\lambda} \phi_{\Lambda \lambda}^{*} .
$$

Since for $S=0$ the helicity structure of Fig. 3 corresponds to a factorized form - the product of two independently varying $\phi$ functions - and, as shown in Eq.(11), these two components transform under Parity independently from one another, The following relations hold between the chiral odd amplitudes and the chiral even ones for $S=0$,

$$
\begin{aligned}
& A_{++,--}^{(0)}=A_{++,++}^{(0)} \\
& A_{++,+-}^{(0)}=-A_{++,-+}^{(0) *} \\
& A_{+-,++}^{(0)}=-A_{-+,++}^{(0) *},
\end{aligned}
$$

Notice that these relations are valid only if one of the two $\phi$ functions is real. By using Parity symmetry one cannot connect directly the chiral odd amplitude $A_{+-,-+}$, with its chiral even counterpart $A_{+-,+-}$since both involve complex $\phi$ functions. Physically this corresponds to the fact that $A_{+-,-+}$involves a double spin flip, and it must therefore be proportional to $\Delta_{\perp}^{2}=\left(t_{0}-t\right)(1-\zeta)$, while $A_{+-,+-}$is non-flip.

In Fig. 8 we show both the chiral even GPDs (left panel) and the chiral odd GPDs (right panel) evaluated using the model described in this paper at $\zeta=0, t=0$, plotted vs. $X$ at fixed $Q^{2}=2 \mathrm{GeV}^{2}$. The chiral even GPDs were already evaluated in Ref.[2] by using the recursive fitting procedure described above. Notice that as

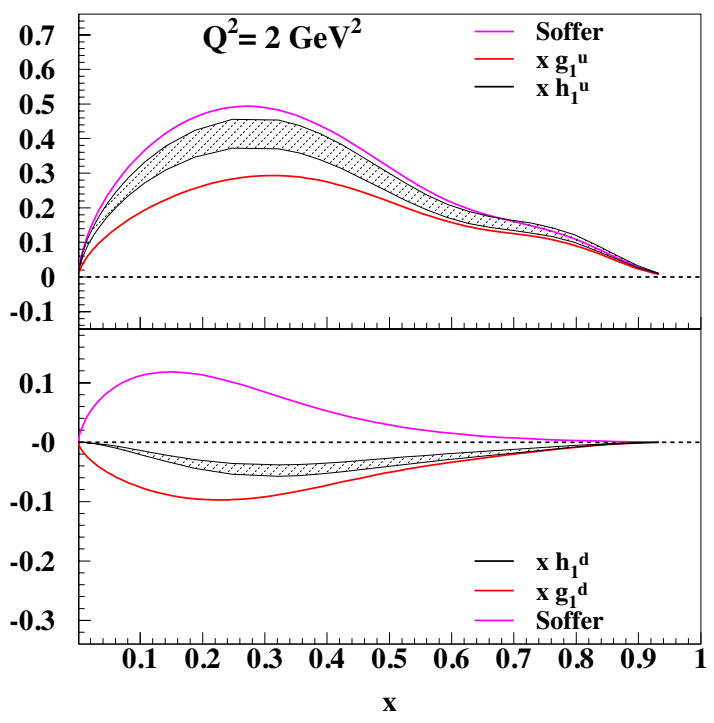

Figure 9. The transversity function, $h_{1}\left(x, Q^{2}\right) \equiv \widetilde{H}_{T}\left(X, 0,0, Q^{2}\right)$ plotted along with theoretical errors (hashed area) for the up (top panel) and down (bottom panel) quarks. The other curves in the figure represent the Soffer bound on the magnitude of $h_{1}$, and the values of $g_{1}^{u, d}$, respectively. Adapted from Ref. [4].

a byproduct of our analysis we obtain an independent extraction of, $H_{T}^{q}\left(X, 0,0 ; Q^{2}\right) \equiv h_{1}^{q}\left(X, Q^{2}\right)$ (upper panels). In Fig.9 we show transversity in more detail, compared with $g_{1}^{q}(X)$, and the Soffer bound, $f_{1}(X)+g_{1}(X)$. It is interesting to notice how, from exclusive pseudoscalar electroproduction data, we obtain an independent extraction of this quantity.

In Fig. 11 we show the predicted [8] "transverse spin-flavor dipole moments", defined by Burkardt as the transversity analog of the anomalous magnetic dipole moments [20].

Finally we consider the observable cross sections for exclusive $\pi^{0}$ electroproduction. The various GPDs calculated for the chiral odd sector enter the cross section terms for $\pi^{0}$ electroproduction, which, using the notation of Ref.[21] (based on [22]), can be defined as,

$$
\begin{gathered}
\frac{d^{4} \sigma}{d x_{B j} d y d \phi d t} \\
=\Gamma\left[F_{U U, T}+\epsilon F_{U U, L}+\epsilon \cos 2 \phi F_{U U}^{\cos 2 \phi}\right. \\
\left.\quad+\sqrt{\epsilon(\epsilon+1)} \cos \phi F_{U U}^{\cos \phi} \ldots\right]
\end{gathered}
$$

where we leave out all the polarized beam or target terms for brevity.

In Fig. 12 we show the unpolarized cross section components for the kinematics $x_{B j}=0.13, Q^{2}=1.2 \mathrm{GeV}^{2}$. Other kinematics, along with various asymmetries can be seen in Ref. [4, 8]. Overall, the agreement with data is notable. 

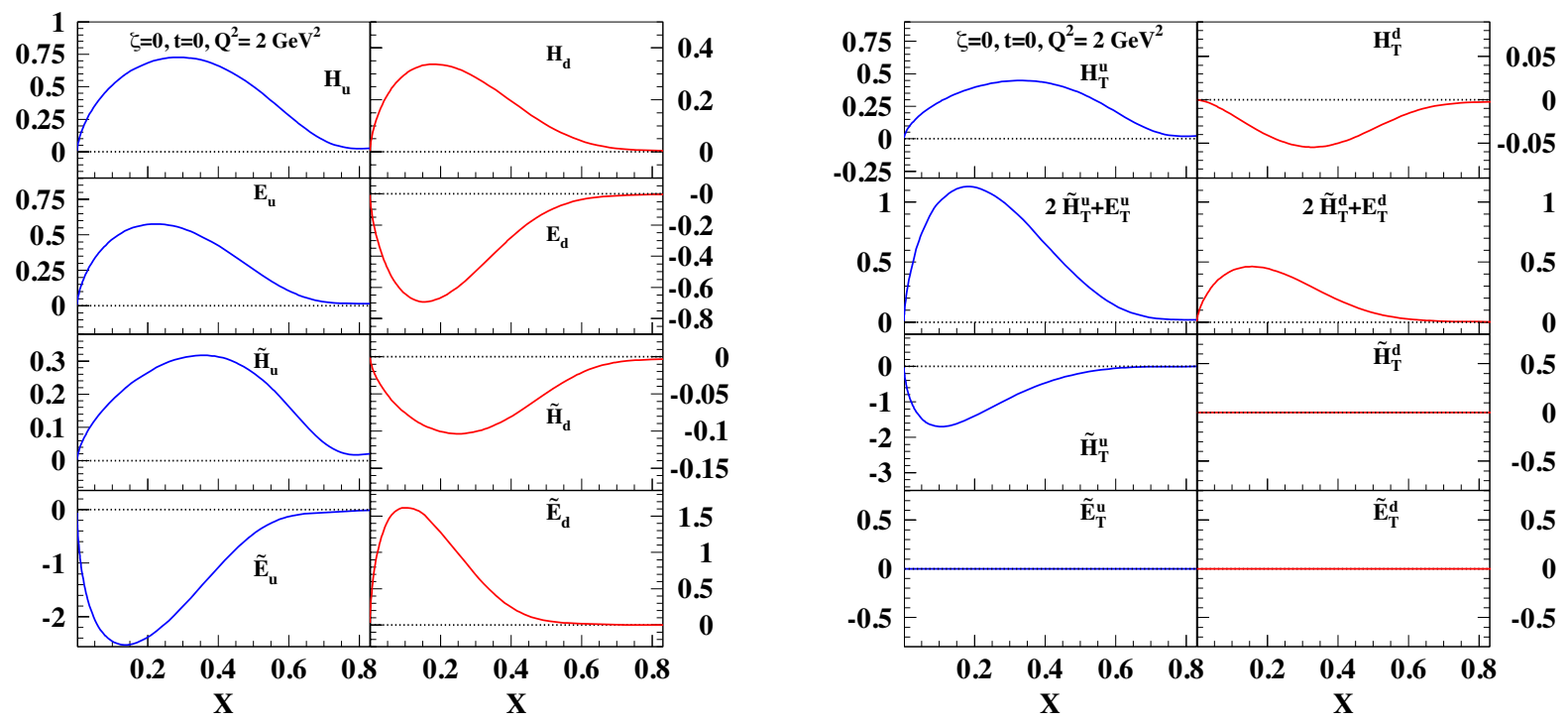

Figure 8. The chiral even (left panel) and chiral odd GPDs (right panel) evaluated using the model described in the text at $\zeta=0, t=0$, plotted vs. $X$ at fixed $Q^{2}=2 \mathrm{GeV}^{2}$. Adapted from Ref. [4]
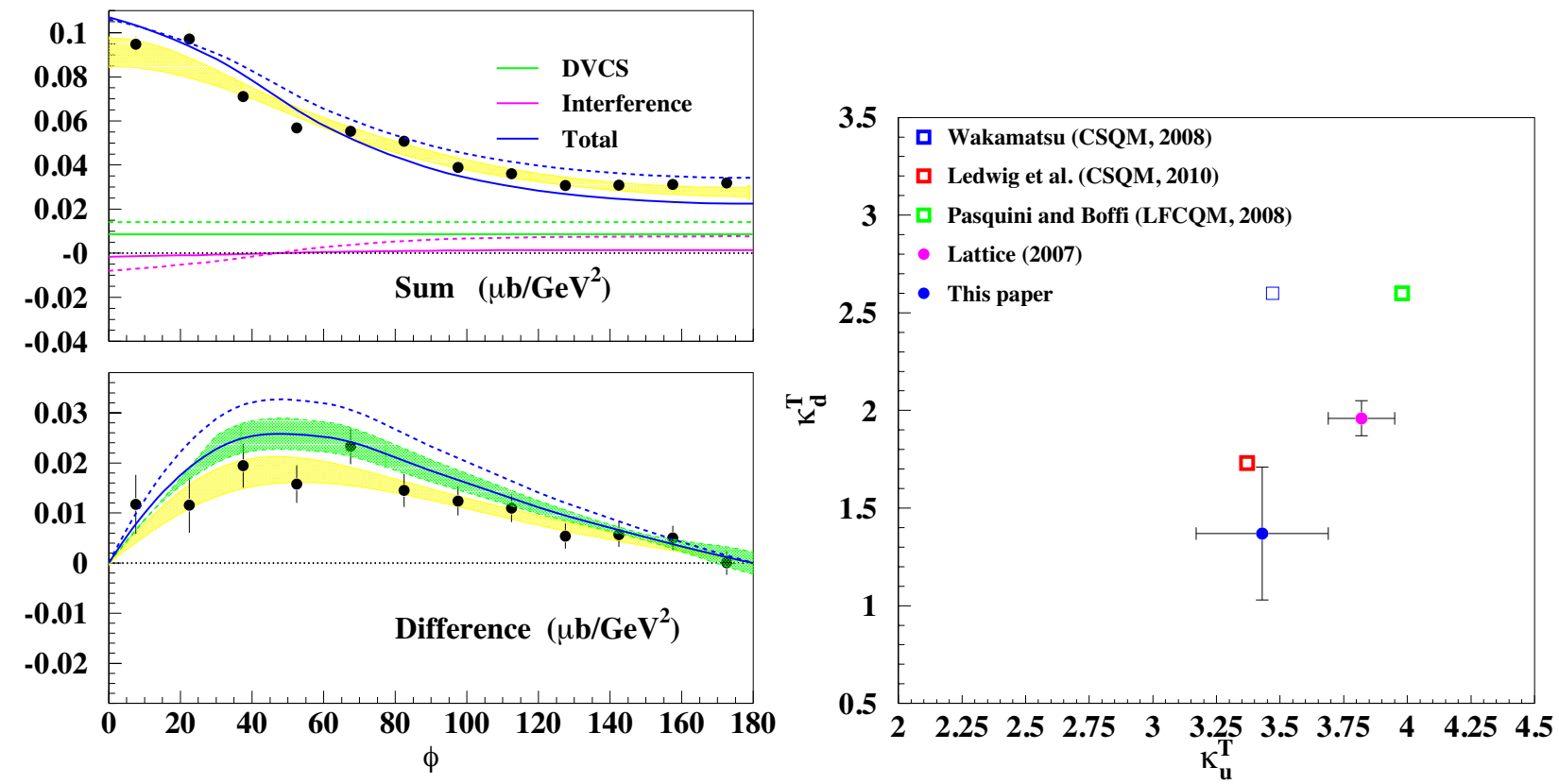

Figure 10. HallA data [23] for the "sum" (upper panel) and "difference" (lower panel) of the two electron beam polarizations. Shown are curves including the contribution of the $\zeta$ dependent factor from Eq.(4) (full lines), and neglecting it (dashed lines). Adapted from Ref. [2].

\section{Acknowledgements}

We thank the organizers of Transversity 2014 for a productive workshop in an excellent setting. This work was funded in part by U.S. DOE grant DE-FG02-01ER4120 (S.L.).
Figure 11. Predictions and determinations of "transverse spinflavor dipole moments" $\kappa_{u}^{T}, \kappa_{d}^{T}$. Adapted from Ref. [8].

\section{References}

[1] A. Courtoy, G. R. Goldstein, J. O. G. Hernandez, S. Liuti and A. Rajan, Phys. Lett. B 731 (2014) 141.

[2] G. R. Goldstein, J. O. Hernandez and S. Liuti, Phys. Rev. D 84, 034007 (2011).

[3] J. O. Gonzalez-Hernandez, S. Liuti, G. R. Goldstein and K. Kathuria, Phys. Rev. C 88, 065206 (2013) 

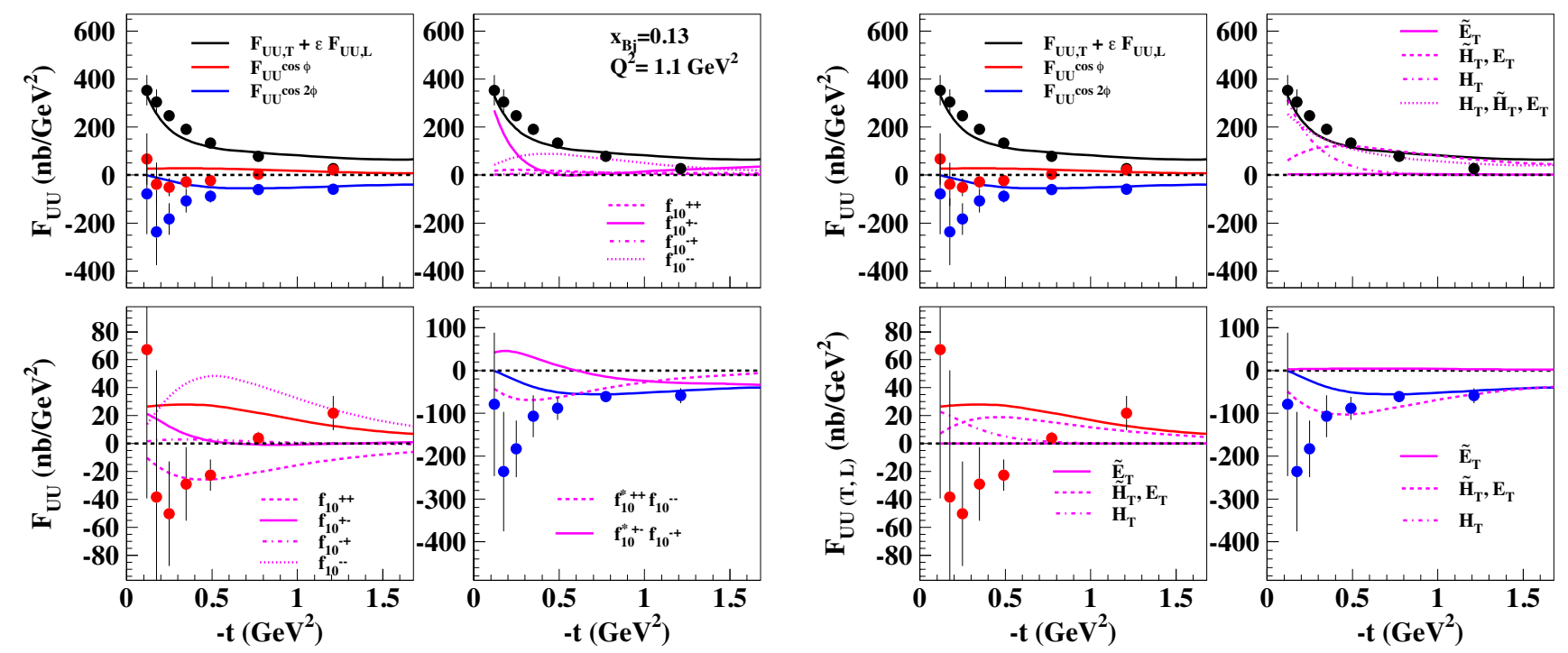

Figure 12. LEFT: Unpolarized cross section components, $F_{U U, T}+\epsilon F_{U U, L}, F_{U U}^{\cos 2 \phi}$, and $F_{U U}^{\cos \phi}$ in the kinematical bin, $x_{B j}=0.13, Q^{2}=1.2$ $\mathrm{GeV}^{2}$. The upper left panel shows all components along with the data from Ref.[24]. The other panels show the contributions from the various helicity amplitudes. Adapted from Ref. [4].

[4] G. R. Goldstein, J. O. Hernandez and S. Liuti, [arXiv:1311.0483 [hep-ph]]

[5] R.L. Jaffe and X. Ji, Phys. Rev. Lett. 67, 552 (1991), and references contained therein.

[6] G.R. Goldstein and M.J. Moravcsik Ann. Phys. (N.Y.) 98, 128 (1976); 142, 219 (1982); 195, 213 (1989).

[7] J. Ralston and D.E. Soper, Nucl. Phys. B 152, 109 (1979).

[8] G. R. Goldstein, J. O. Hernandez and S. Liuti, submitted Phys.Rev.D, [arXiv:1401.0438 [hep-ph]].

[9] E. Leader, These Proceedings.

[10] X. -D. Ji, Phys. Rev. Lett. 78, 610 (1997).

[11] D. V. Kiptily and M. V. Polyakov, Eur. Phys. J. C 37 (2004) 105.

[12] A.V. Belitsky, D. Mueller and A. Kirchner, Nucl. Phys. B 269, 323 (2002).

[13] A. Airapetian et al. [HERMES Collaboration], JHEP 1006, 019 (2010)

[14] P.V. Landshoff, J.C. Polkinghorne and R.D. Short, Nucl. Phys. B 28, 225 (1971).
[15] S. J. Brodsky, F. E. Close and J. F. Gunion, Phys. Rev. D 5, 1384 (1972).

[16] S. Ahmad, H. Honkanen, S. Liuti, S.K. Taneja, Phys. Rev. D75, 094003 (2007), ibid, Eur. Phys. J. C63, 407421 (2009).

[17] A. Airapetian et al. [ HERMES Collaboration ], JHEP 0806, 066 (2008).

[18] A. Airapetian et al. [ HERMES Collaboration ], JHEP 0911, 083 (2009).

[19] G. D. Cates, C. W. de Jager, S. Riordan and B. Wojtsekhowski, Phys. Rev. Lett. 106, 252003 (2011).

[20] M. Burkardt, Phys. Rev. D 72, 094020 (2005).

[21] A. Bacchetta, M. Diehl, K. Goeke, A. Metz, P. J. Mulders and M. Schlegel, JHEP 0702, 093 (2007)

[22] M. Diehl and S. Sapeta, Eur. Phys. J. C 41, 515 (2005)

[23] C. Munoz Camacho et al. [ Jefferson Lab Hall A and Hall A DVCS Collaborations ], Phys. Rev. Lett. 97, 262002 (2006).

[24] I. Bedlinskiy, et al., Phys.Rev.Lett. 109, 112001(2012). 